\title{
Spike Width Reduction Modifies the Dynamics of Short- Term Depression at a Central Synapse in the Locust
}

\author{
J. E. Niven and M. Burrows \\ Department of Zoology, University of Cambridge, Cambridge CB2 3EJ, United Kingdom
}

Short-term synaptic depression is an important component of computation within neural networks, but little is known of its contribution to information processing during synaptically generated spike trains. We analyzed short-term synaptic depression at a synapse between two identified motoneurons innervating the hind leg of the locust: the FETi-FITi synapse (fast extensor tibiae-flexor tibiae). Brief electrical stimulation of a single hind leg proprioceptor, the lump receptor (LR), led to prolonged sequences of spikes in FETi, similar in number and frequency to those during natural kicking movements. Depression at the FETi-FITi synapse during LR-evoked spike bursts was compared quantitatively to that during antidromic spike trains evoked by electrical stimulation of FETi in the extensor tibiae muscle, and by modeling. The magnitude of the short-term depression was significantly greater during LR-evoked spike trains. On the basis of the model parameters required to fit the depression, the FETi-FlTi synapse is predominantly used for transmitting the timing of the onset of FETi spiking rather than its spike rate. During LR-evoked spike trains, there was a rapid reduction in presynaptic spike width that did not occur during antidromic spike trains under physiological calcium concentrations. This produced a concomitant reduction in the amplitude of the FITi EPSP, suggesting that it contributed to the differences between the two stimulation regimes. Differences in the short-term depression between synaptically evoked and antidromic spike trains emphasize that the properties of synaptic information transfer are dependent on the in vivo conditions at the synapse and may not be reproduced by in vitro spike trains.

Key words: synaptic depression; motor control; proprioception; Schistocerca gregaria; lump receptor; presynaptic waveform; presynaptic inhibition

\section{Introduction}

Many chemical synapses undergo short-term frequencydependent changes in their efficacy, resulting in depression or facilitation (Eccles et al., 1941; Feng, 1941; Zucker, 1989). Changes in synaptic efficacy influence the information transferred between neurons and contribute to cortical processing (Thomson and Deuchars, 1994; Abbott et al., 1997; Tsodyks and Markram, 1997), motor pattern generation (Combes et al., 1999; Parker, 2000), and muscle activity (Del Castillo and Katz, 1954; Sen et al., 1996). This form of synaptic plasticity is often studied in vitro in pairs of cells by generating constant frequency spike trains in the presynaptic cell and recording the postsynaptic response. Although this approach is useful for examining the mechanisms underlying synaptic plasticity, these properties may not be congruent with those of the in vivo synapses. During natural spike trains in vivo, the presynaptic and postsynaptic neurons receive synaptic inputs that may profoundly alter the efficacy of a synaptic connection (e.g., presynaptic inhibition). How do these synaptic inputs interact with frequency-dependent mechanisms to determine the efficacy of a synapse?

A monosynaptic connection exists between the fast motoneu-

Received Feb. 11, 2003; revised May 27, 2003; accepted June 13, 2003.

This work was supported by a Biotechnology and Biological Sciences Research Council Studentship (J.E.N.) and a Welcome Trust Research Grant (M.B.). We thank H. Robinson, D. Parker, I. Kleppe, and B. Hedwig for discussions and comments on a previous version of this manuscript.

Correspondence should be addressed to Dr. J. E. Niven, Department of Zoology, Downing Street, University of Cambridge, Cambridge CB2 3EJ, UK. E-mail: jen22@hermes.cam.ac.uk.

Copyright $\odot 2003$ Society for Neuroscience $\quad$ 2270-6474/03/237461-09\$15.00/0 ron (FETi) innervating the extensor tibiae muscle of the hind leg of the locust and the flexor motoneurons (FlTi) innervating the antagonistic flexor tibiae muscle (Hoyle and Burrows, 1973; Burrows et al., 1989). During antidromic spike trains, this synapse undergoes frequency-dependent depression (Parker, 1995). A reduction of the FETi spike height and width correlates with depression in the amplitude of the FlTi EPSP (Parker, 1995). At several synapses, the presynaptic spike waveform influences the opening of voltage-activated $\mathrm{Ca}^{2+}$ channels and, hence, vesicle release at the presynaptic terminal, enabling changes in it to contribute to short-term synaptic depression (Siegelbaum et al., 1982; Wheeler et al., 1996; Sabatini and Regehr, 1997).

Stimulation of a particular joint proprioceptor, the lump receptor (LR) (Heitler and Burrows, 1977), triggers bursts of spikes in FETi closely resembling its activity (spike number and frequency) during kicking, but does not evoke synaptic inputs in a subpopulation of FlTi motoneurons. The lack of synaptic inputs to the FlTi motoneurons enabled a comparison of depression at the FETi-FlTi synapse generated by antidromic spike trains with that generated by LR-evoked spike trains during which spikes are evoked by synaptic inputs to FETi. Synaptic depression was simulated during LR-evoked spike trains and used to predict depression under constant frequency antidromic spike trains using a model in which the probability of neurotransmitter release is updated at each spike (Tsodyks and Markram, 1997; Markram et al., 1998; Finnerty et al., 1999). The dynamics of short-term depression during LR-evoked and antidromic spike trains was markedly different, and model parameters obtained under one 

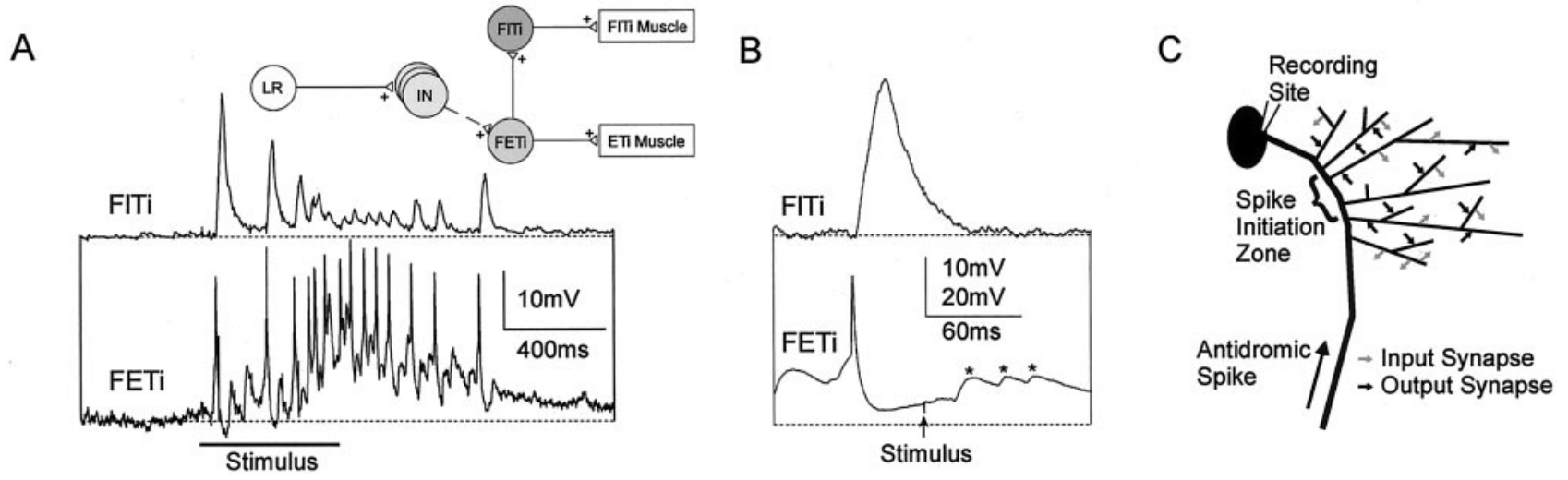

Figure 1. Depression at theFETi-FITicentral synapse during synaptically evoked spike trains. $A$, Brief electrical stimuli applied to the LNevokes bursts of spikes in the FETithat continue after the termination of the stimuli. Spikes in FETi evoke an EPSP in antagonistic FITi via a monosynaptic connection. Inset, A schematic of the circuit generating the bursts of spikes in FETi and their corresponding FITi EPSPs. B, A single FETi spike evokes a monosynaptic EPSP in a FITi. Stimulation of the LN evokes EPSPs in FETi (indicated by asterisk) that evoke spikes but does not evoke inputs to a subset of FITimotoneurons, which receive inputs only from the FETiand not from local interneurons. C, A schematic diagram of the relative positions of input (black arrows) and output (gray arrows) synapses in FETi. Dashed lines indicate the resting potential in all traces.

stimulation regime could not predict the depression during the other regime, suggesting that synaptic inputs to FETi modify the dynamics of depression at this synapse. Such modification of depression/facilitation by presynaptic inputs may be widespread in neurons, preventing the prediction of in vivo synaptic properties from artificially generated spike trains.

\section{Materials and Methods}

Preparation and electrophysiology. Adult desert locusts (Schistocerca gregaria; Forskål) of either sex were taken from a crowded colony maintained in the Department of Zoology, University of Cambridge. They were mounted ventral surface uppermost in modeling clay (Plasticine), and the femur and tibia of each leg was fully restrained, with the exception of the tibia of the left hind leg, which was able to extend fully. During experiments in which the lateral nerve (LN), or one of its branches, which contain the axons of the LR, was stimulated, the tibia was not allowed to flex to angles of $<20^{\circ}$ to prevent damage to stimulation electrodes. The metathoracic ganglion was exposed by cutting a window in the ventral thorax and removing surrounding air sacs while leaving the main trachae intact. The mesothoracic and metathoracic ganglia were stabilized on a wax-coated silver platform to prevent movement during the experiment. The thorax was continually perfused with physiological saline (Usherwood and Grundfest, 1965$)$ at room temperature $\left(22-24^{\circ} \mathrm{C}\right)$. The connectives linking the metathoracic ganglion to the mesothoracic and fourth abdominal ganglia and peripheral nerves were cut to prevent descending, ascending, and peripheral inputs from modulating depression at the FETi-FlTi (fast extensor tibiae-flexor tibiae) synapse. Nerve 5 branch B2 and branch B1, proximal to the innervation of the extensor muscle, remained intact, allowing the identification and stimulation of the motoneurons studied here. The sheath of the ganglion was treated with protease (Sigma type XIV) for $\sim 45$ sec to facilitate penetration with thick-walled borosilicate glass microelectrodes $(40-80 \mathrm{M} \Omega$ ) filled with $2 \mathrm{M}$ potassium acetate.

A window was cut into the ventral posterior surface of the distal femur to reveal the LN, which was recorded or stimulated extracellularly with two $100 \mu \mathrm{m}$ silver hook electrodes. The electrodes were placed either on the whole nerve or on the nerve branch innervating only the lump receptor (LRN). This allowed the LR to be stimulated independently of other F-T joint proprioceptors. Electrical stimulation (0.5-1 msec; $120 \%$ of threshold) of the $\mathrm{LN}$ or $\mathrm{LRN}$ at $7 \mathrm{~Hz}$ or more (up to $50 \mathrm{~Hz}$ ) reliably evoked bursts of spikes in FETi that continue beyond the duration of the stimulation. Stimulation was stopped once a burst of spikes was evoked, and sequential bursts were evoked at least 2 min apart to enable the FETi-FlTi synapse to recover fully after a spike burst.

Intracellular recordings were made from motoneurons controlling tibial extension and flexion, which were identified in the following ways: electrical stimulation of the extensor muscle with a pair of $50 \mu \mathrm{m}$ steel wires excited the peripheral terminals of FETi and evoked an antidromic spike. Flexor motoneurons were identified by a short latency monosynaptic EPSP that followed each antidromic FETi spike (Hoyle and Burrows, 1973; Heitler and Burrows, 1977). Individual flexor motoneurons were identified according the criteria described in Parker (1995). Up to three flexor motoneurons were recorded from a single animal.

Intracellular recordings were stored on a Racal FM tape recorder and were subsequently analyzed offline using a Cambridge Electronic Design A/D conversion interface and Spike 2 software. All statistical tests were performed using MINITAB and Microsoft Excel. The results are based on 46 recordings from 33 animals. Here, $\mathrm{N}$ refers to the number of animals used and $n$ to the number of repeats within a single animal.

Analysis and modeling. The model used to characterize depression at the FETi-FlTi synapse was based on the three-parameter model proposed by Tsodyks and Markram (1997): (1) the absolute response if all transmitter at the synapse was released $(A) ;(2)$ the fraction of available transmitter that is used after a spike $(U)$; and (3) the time course of recovery at the synapse between spikes $\left(\tau_{\mathrm{Rec}}\right)$. The model is formulated so that after a spike, a fraction $(U)$ of the absolute amount of transmitter available $(A)$ is released that evokes an EPSP in the postsynaptic neuron. The released transmitter is unavailable to subsequent spikes and recovers with a time constant of $\tau_{\text {Rec }}$.

Additional parameters were added to determine any possible effects of underlying facilitation on the depression at the FETi-FlTi synapse. $U$ applies only to the first spike in a train, and $u$ is the updated value of $U$. The updated value of $u$ incorporates facilitation at the synapse via a single exponential, $\tau_{\text {facil }}$, by which $u$ decays to its starting value $U . R$ is the fraction of total remaining transmitter available for release when any spike occurs.

The transmitter released after the first spike is $A^{\star} U$ and $\mathrm{R}_{0}=1$, because the maximum amount of transmitter is stored before the spike. Immediately after this spike, the remaining transmitter is $r=1-U$, and at the occurrence of the next spike, the remaining transmitter will be this plus the amount of transmitter recovered. Hence, the amount of transmitter remaining is dependent on the timing of subsequent spikes and is:

$$
R_{n+1}=R_{n}\left(1-u_{n+1}\right) \exp \left(\frac{-\Delta t}{\tau_{\mathrm{Rec}}}\right)+1-\exp \left(\frac{-\Delta t}{\tau_{\mathrm{Rec}}}\right)
$$

where $\Delta \mathrm{t}$ is the time interval between the $\mathrm{nth}$ and $(\mathrm{n}+1)$ th AP where:

$$
u_{n+1}=u_{n} \exp \left(\frac{-\Delta t}{\tau_{\text {facil }}}\right)+U\left(1-u_{n} \exp \left(\frac{-\Delta t}{\tau_{\text {facil }}}\right)\right)
$$

During spike trains in which there is no contribution from facilitation $\tau_{\text {facil }}$ approaches 0 and the model simplifies to $u_{n}=U$ (for review, see Tsodyks and Markram, 1997; Markram et al., 1998).

Data fitting. Only FlTi motoneurons in which there were no discernable synaptic inputs (other than those inputs from FETi) during LR 
$\mathrm{Ai}$

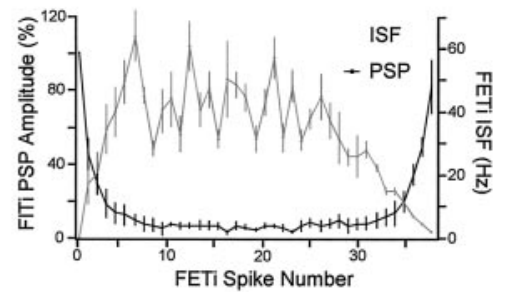

B

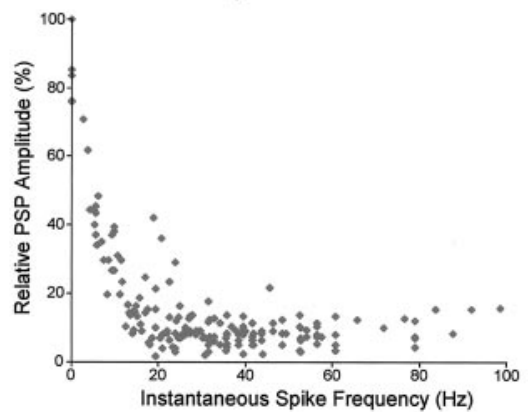

Aii

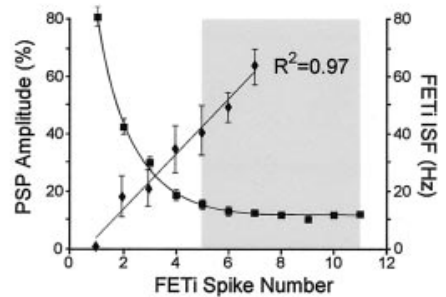

C

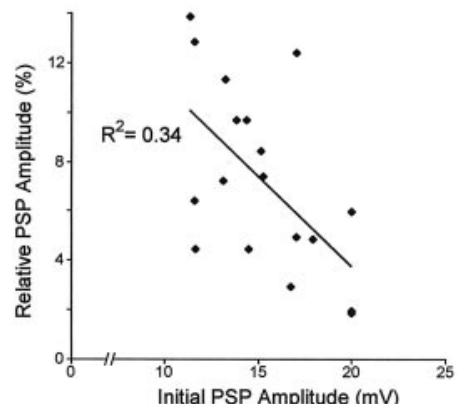

Figure 2. The dynamics of depression at the FETi-FITi synapse during natural-like synaptically evoked spike trains. Ai, The FETi ISF and posterior fast FITi EPSP amplitude corresponding to each spike during a burst. Aii, The mean ( \pm SE) of 19 FFITi motoneuron EPSPS during bursts of spikes in FETi fitted by a single exponential. The shaded area indicates the plateau region of the FETi ISF. $B$, The relationship between the FETi ISF and FITi EPSP amplitude for a single FETi-FITi pair $(n=6)$ could be fitted by a single exponential decay. C, The relationship between depression at the FETi-FITisynapse and the initial FITi EPSP amplitude. The level of depression after 15 spikes was dependent on the initial FITi EPSP amplitude; depression was greater after larger initial EPSPs than after smaller initial EPSPS.

stimulation were used for analysis. EPSP amplitudes in all FlTi motoneurons were measured from the baseline to peak, reducing the trains of EPSPs to a series of amplitudes and times. Similarly, AP amplitude was measured from the baseline to peak, and its width at half height. Experimentally derived data were fitted by iterating the parameters $(A, U$, and $\tau_{\text {Rec }}$ ) of the basic model (i.e., $\left.u_{n}=U\right)$ and minimizing $\left(E P S P_{\text {Exp. }}-\right.$ $\left.E P S P_{\text {Model }}\right)^{2}$ for all EPSPs in a spike train. The additional parameters enabling facilitation were then introduced, and new values were calculated for $A, U$, and $\tau_{\text {Rec }}$. The starting parameters for the model were chosen at random, and the model was iterated to produce a global minimum. The fit of the model to the data and its ability to predict novel spike trains was assessed by comparing the standard error of the estimate divided by the mean (a unitless measure) for each fit. This method is more reliable than the SE of the estimate when comparing regressions (Zar, 1996).

\section{Results}

\section{Depression and recovery at the FETi-FlTi synapse during natural spike trains}

Electrical stimulation of the nerve innervating the LR evoked prolonged spike trains in the single FETi that outlasted the duration of the stimulus (Fig. $1 A$ ). Simultaneous recordings of FETi and FlTi motoneurons showed that some FlTi motoneurons did not receive synaptic inputs after electrical stimulation of the LR (Fig. 1B). The LR-evoked FETi spike trains contained similar numbers of spikes with a similar frequency distribution to those occurring during kicking. The bursts typically lasted for 1-4 sec, with each burst consisting of 10-40 spikes at frequencies up to 70 $\mathrm{Hz}$ (cf. Burrows, 1995). Each FETi spike was followed by an EPSP in FlTi motoneurons transmitted by a monosynaptic connection (Fig. $1 A, B$ ). The central output synapses of FETi are intermingled with its input synapses on neuropilar processes that branch from the primary neurite (Watson and Burrows, 1981; Burrows et al., 1989). At this region, there is a transition between active and inactive membrane along the primary neurite, and spike initiation takes place (Gwilliam and Burrows, 1980) (Fig. 1C).
During LR-evoked spike trains, the amplitude of the FITi EPSPs depressed to $~ 6 \%$ $(6.43 \pm 0.65 \% ; \mathrm{N}=19 ; n=6$; mean $\pm \mathrm{SE}$; where $\mathrm{N}$ refers to the number of animals and $n$ to the number of repeats per animal) of their initial amplitude (Figs. 1A, 2Ai). The depression at the FETi-FlTi synapse was fitted by a single exponential decay with a rate constant of $1.39 \mathrm{sec}^{-1}$ (Fig. 2Aii). Most of this depression occurred over the initial four to six spikes, during which the instantaneous spike frequency (ISF) of FETi increased linearly before reaching a plateau phase of $\sim 40 \mathrm{~Hz}$ on which fluctuations of up to $30 \mathrm{~Hz}$ were superimposed (Fig. 2Ai,Aii). These fluctuations had little effect on the amplitude of the FlTi EPSP (Fig. 2Ai). At the end of this plateau phase, the FETi spike frequency slowed, allowing recovery in the EPSP amplitude within $1 \mathrm{sec}$ (Fig. 2Ai).

\section{Frequency dependence of depression at the FETi-FlTi synapse}

Figure $2 B$ shows the relationship between ISF and the FlTi EPSP amplitude for three successive spike trains recorded from the a single FETi-FlTi pair. During the first four to six spikes (i.e., before the onset of the plateau phase), the frequency of FETi spikes accurately predicted the EPSP amplitude; spikes with similar ISFs occurred at similar times within each spike train, and they, therefore, contained information about the preceding number of spikes in the train and their interspike intervals. Once the ISF had plateaued, there was little or no further depression in the FlTi EPSP, and large fluctuations in the FETi ISF had little effect on EPSP amplitude (indicated by the flattening of the exponential decay in Fig. $2 B$ ).

\section{Depression at the FETi-FlTi synapse is dependent on the initial EPSP amplitude}

Synaptic depression is often generated by presynaptic mechanisms that are affected by changes in the initial release probability (Zucker, 1989). If depression at the FETi-FlTi synapse is produced by a presynaptic mechanism, the final level of depression may be dependent on the amplitude of the first EPSP in a train. Larger initial EPSPs may indicate that more of the available synaptic vesicles have been released. The amplitude of the first FlTi EPSP varied from $11.1-20.5 \mathrm{mV}(\mathrm{N}=18 ; n=6)$, a similar range to FlTi EPSPs evoked by antidromic spikes in FETi (Burrows et al., 1989; Parker, 1995). During bursts of spikes with larger initial EPSP amplitudes, the final level of depression was greater $(p<$ 0.05 ; $t$ test) (Fig. $2 C)$. The relationship was, however, weak $\left(r^{2}=\right.$ 0.34 ) possibly because of variation in the spiking pattern during the burst. During antidromic spiking at this synapse, the final level of depression was also dependent on the initial EPSP amplitude (Parker, 1995).

\section{Comparison with depression during antidromic trains of spikes in FETi}

Despite the differences in the waveform of antidromic and synaptically generated spikes in FETi (Gwilliam and Burrows, 1979), they evoked EPSPs with similar amplitudes and time courses in FlTi motoneurons (Fig. 3A). During antidromic stimulation at 
$\mathrm{Ai}$

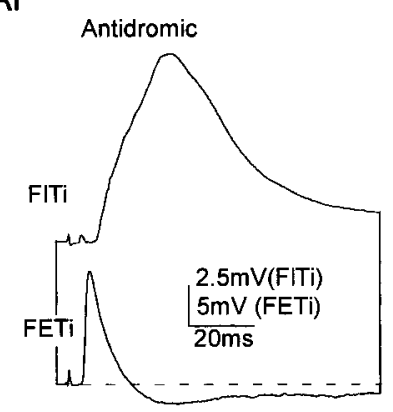

B

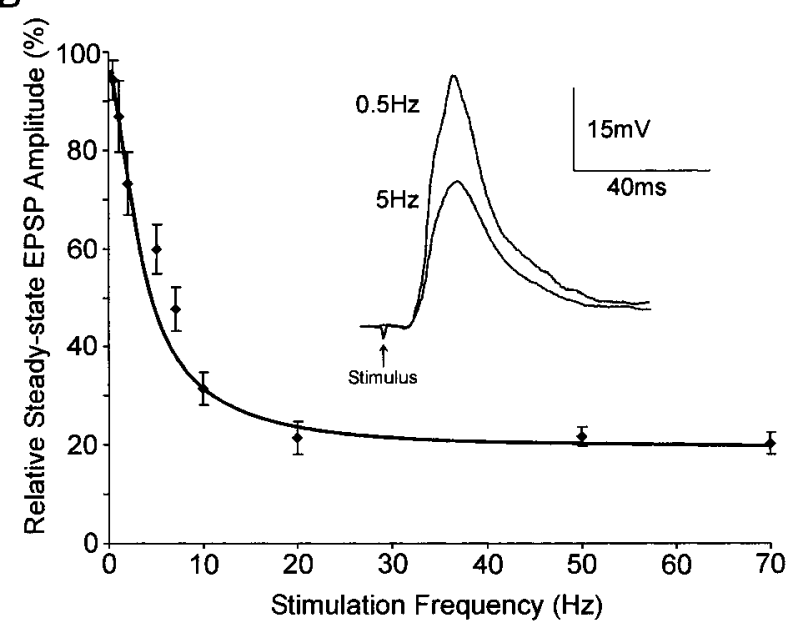

Figure 3. Depression at the FETi-FITi synapse during antidromically generated spike trains. $A$, EPSPs evoked in FITi motoneurons have similar amplitudes and durations after antidromic spikes (left) and synaptically generated spikes (right) in FETi. B, Steady-state EPSP amplitudes after depression at the FETi-FITi synapse during antidromic spike trains in FETi calculated after the first 10 spikes (mean $\pm S D ; N=12 ; n=10$ ) fitted with a single exponential. Inset, Mean steady-state EPSP amplitudes at 0.5 and $5 \mathrm{~Hz}$ from a single FITi motoneuron $(n=10)$.

frequencies up to $70 \mathrm{~Hz}$, the maximum spike frequency that occurred during LR-evoked spike trains, the level of depression at the FETi-FlTi synapse did not drop below 20\% (20.36 $\pm 0.33 \%$; $\mathrm{N}=19 ; n=10$; mean $\pm \mathrm{SE}$ ) of the initial amplitude (Fig. $3 B$ ). The relationship between the antidromic spike frequency and the steady-state EPSP amplitude, at which there is no further reduction in EPSP amplitude, was fitted by a single exponential with a rate constant of $6.21 \mathrm{sec}^{-1}$. Depression during antidromic spike trains never exceeded depression during synaptically generated spike trains, even when the frequency of the antidromic spikes was greater than the maximum frequency of synaptically generated spikes.

\section{Modeling short-term depression at the FETi-FITi synapse}

A model was used to compare the dynamics of depression at the FETi-FlTi synapse during synaptically and antidromically generated spike trains. The model produced an accurate fit of the depression at the FETi-FlTi synapse during a synaptically driven FETi spike burst $(\mathrm{N}=1 ; n=6$ ) (Fig. $4 A$ ), shown by plotting the observed against the predicted EPSP amplitudes (Fig. $4 B$ ). The mean error in the fit of the model to LR-evoked spike trains was $0.16 \pm 0.06(\mathrm{~N}=19 ; n=6$; for details of the calculation of the mean error, see Materials and Methods). To ensure that a more accurate fit could not be obtained from the model using additional parameters, a facilitation time constant was added. This produced only a small improvement in the fit $(0.004 \pm 0.0004)$
(Fig. $4 C, D$ ), suggesting that any underlying facilitating mechanisms make only a small contribution.

The model was tested further by producing predictions to novel spike trains (individual spike trains) (Fig. $4 E, F$ ). Errors between the model predictions of novel LR-evoked spike trains and the observed EPSP amplitudes were greater than the errors in the fit of the model to specific data sets, although this increase in the error was small $(0.05 \pm 0.02)$ and fell within the variation in the fitted model spike trains (Fig. $4 E$, F, compare with Fig. $6 B, D$ ).

For constant frequency antidromic spike trains, the model consistently predicted a faster rate of depression and a smaller EPSP amplitude for a given spike frequency than was observed (Fig. 5A, B). There was a significant increase in the mean error of model predictions for antidromic spike trains at frequencies above $10 \mathrm{~Hz}(0.99 \pm 0.14 ; p<0.001 ; \mathrm{N}=17 ; n=10)$. To test whether the model could accurately fit the antidromic data, both with and without the additional facilitation parameter, it was allowed to iteratively fit the antidromic data (Fig. $5 C-F$ ). The model was then able to fit the antidromic spike trains, although its parameters were significantly different from those used to fit the LR-evoked spike trains (Fig. 5C,D). The addition of a facilitation parameter, as for the synaptically evoked spike trains, did not affect the fit of the model (Fig. $5 E, F$ ).

The model was able to fit the depression at the FETi-FlTi synapse for all types of FlTi motoneurons observed during simultaneous recordings and during sequential recordings, including
A

C

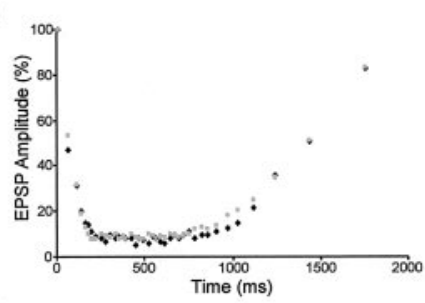

$E$

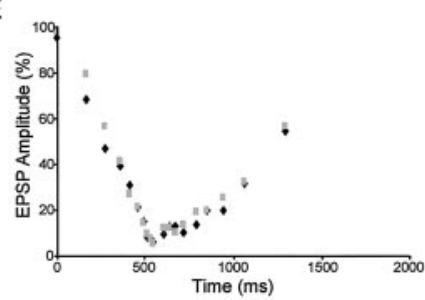

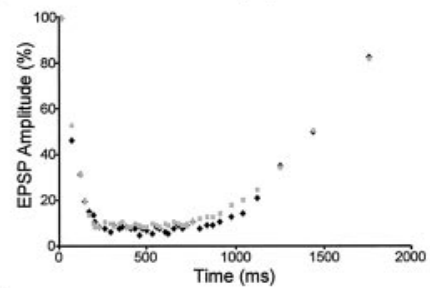

B

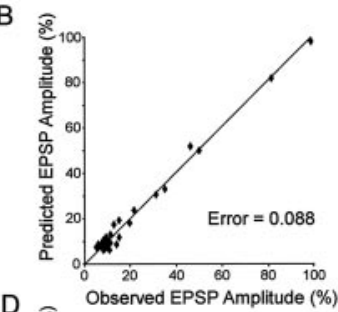

D

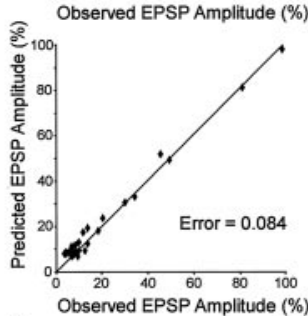

$\mathrm{F}$

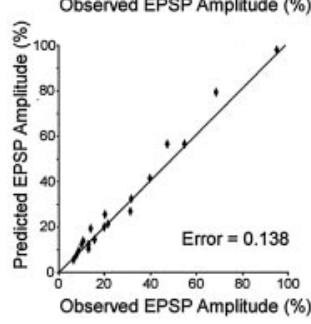

Figure 4. Modeling depression at the FETi-FITi synapse during LR-evoked spike bursts. $A$, Averaged FITi motoneuron responses during FETi spike bursts $(n=6)$ were fitted by a simple three-parameter model of synaptic depression $\left(U=0.61 ; A=159.59 ; \tau_{\text {Rec }}=505.32\right)$. The timing of each spike in a train was measured from the first spike in the train, and the observed (black) and predicted (gray) FITi EPSPs are plotted for each spike. B, Plotting the model against the data show the fit of the model to the data. Points lying on the diagonal indicate a perfect fit. $C$, The addition of a facilitating parameter to the model did not improve the fit of the model to the data $\left(U=0.62 ; A=158.07 ; \tau_{\text {Rec }}=504.02 ; \tau_{\text {Facil }}=13.18\right) . D$, As in $B$. E, The parameters derived from fitting the model to the data enabled the model to accurately predict the responses of the same FITi motoneuron to single FETi spike bursts not used to calculate the original model parameters. $F, A \sin B$. 
A

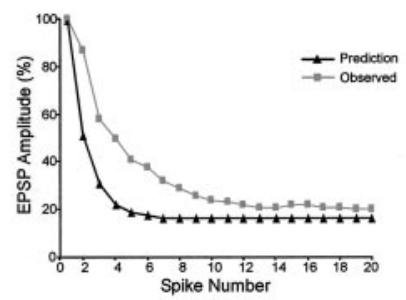

C

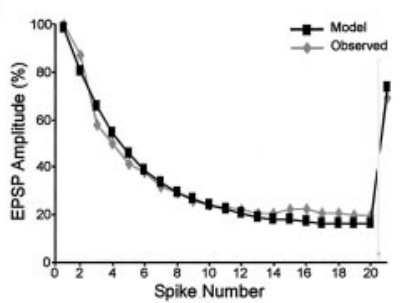

E

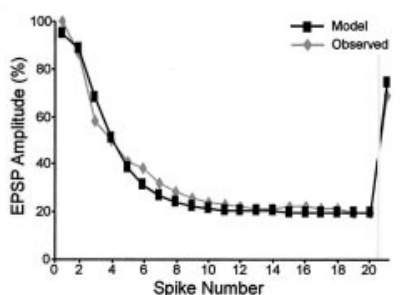

B

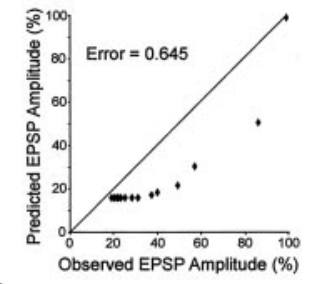

D

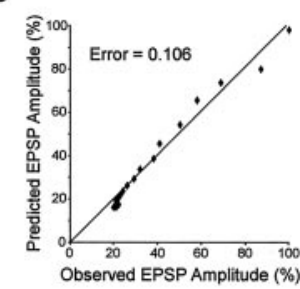

F

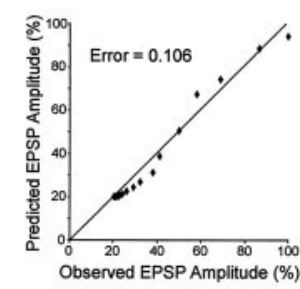

Figure 5. Model predictions do not fit observed antidromic spike trains. $A$, Parameters derived from fitting the model to FITi responses during FETi spike bursts could not predict the responses of the same FITi motoneurons to constant frequency antidromic spike trains at $20 \mathrm{~Hz}$. $B$, Plotting the model against the data show the fit of the model to the data. Points lying on the diagonal indicate a perfect fit. $C$, The model could produce fits to constant frequency antidromic spike trains $\left(U=0.25 ; A=394.31 ; \tau_{\operatorname{Rec}}=1420.70\right)$. The gray bar indicates a 1-sec period of recovery. $D$, As in $B$. $E$, The model with an additional facilitating time constant produced more accurate fits to the constant frequency antidromic spike trains $\left(U=0.43 ; \mathrm{A}=219.90 ; \tau_{\operatorname{Rec}}=\right.$ 1057.71; $\left.\tau_{\text {Facil }}=226.94\right)$. F, As in B.

both fast and intermediate FlTi motoneurons. The model parameters were unique for each FlTi motoneuron, although all of a particular class (e.g., fast FlTi motoneurons) had parameters clustered around similar values. Figure $6 A-D$ shows two FlTi motoneurons recorded sequentially from the same preparation as the FlTi motoneuron in Figure 4. Two (Figs. 4, 6C,D) were fast FlTi motoneurons whereas the third (Fig. $6 A, B$ ) was an intermediate motoneuron. This suggests that, although there is some variation between FlTi motoneurons, the dynamics of depression at the FETi-FlTi synapse occurs within a restricted range.

One prediction made by the model is that if a presynaptic neuron spikes above a certain frequency, known as the limiting frequency, the amplitude of the EPSP evoked by each spike will decrease in proportion to the frequency $(1 / f)$. Therefore, above the limiting frequency, the mean postsynaptic depolarization from the resting potential saturates (i.e., as the spike frequency increases, the EPSP frequency increases but the individual EPSP amplitude decreases). Above the limiting frequency, increases in spike rate do not increase the mean level of depolarization in the postsynaptic neuron. Hence, the limiting frequency sets the frequency above which synapses are unable to transmit information about the presynaptic spike rate (Tsodyks and Markram, 1997). Before reaching this stationary level of mean postsynaptic depolarization, synapses can still transiently affect the level of postsynaptic depolarization. At the FETi-FlTi synapse, the limiting frequency was below $5 \mathrm{~Hz}$, suggesting that it does not transmit information about the FETi spike rate over the majority of the

natural spike frequency range and is more suited to transmitting information about the onset of spiking in FETi (Fig. 7A). Coupled with high values of $U(0.52-0.89)$, this suggests that the FETi-FlTi synapse primarily transmits temporal information about the onset of spiking in FETi (Fig. 7B).

\section{Presynaptic mechanisms}

Changes in the height and width of the FETi spike were measured during synaptically evoked spike trains to determine whether changes in these parameters could account for the increased levels of depression relative to those during antidromic spike trains (Fig. $8 A$ ). The FETi spike height was reduced to $\sim 79 \%$ of its initial height $(78.8 \pm 4.3 \% ; \mathrm{N}=12)$ and was accompanied by a reduction in spike width to $\sim 55 \%$ of the initial width $(54.7 \pm$ $7.6 \%$; $\mathrm{N}=12$ ) (Fig. $8 A, B$ ). These changes in the FETi spike waveform occurred with the same time course as the initial depression of the FITi EPSP (Fig. 8A). Changes in the FETi spike height and width correlate with changes in both the ISF (Fig. $8 C$ ) and FITi EPSP amplitude (Fig. 8D), decreasing spike height and width correlating with depression in the FlTi EPSP and increasing spike height and width correlating with recovery of the EPSP amplitude.

In antidromically induced trains of spikes, there was a smaller reduction in the FETi spike height to $\sim 82 \%(82.1 \pm 3.2 \%$; $\mathrm{N}=$ 12) of the initial height at $70 \mathrm{~Hz}$ (Fig. 9A). However, spike height continued to decrease as the ISF of the antidromic spike trains increased, although there was no accompanying drop in the FlTi EPSP (Fig. 9B, shaded area). In contrast, there was almost no change $(95 \pm 3.4 \% ; \mathrm{N}=12)$ in the spike width during antidromic spike trains at frequencies of up to $70 \mathrm{~Hz}$ (Fig. 9A).

The FETi spike width during the synaptically evoked spike trains correlated strongly with the membrane potential of FETi immediately preceding a spike, increasing membrane potential leading to narrower spikes (Fig. $10 \mathrm{~A}$ ). To test whether changes in
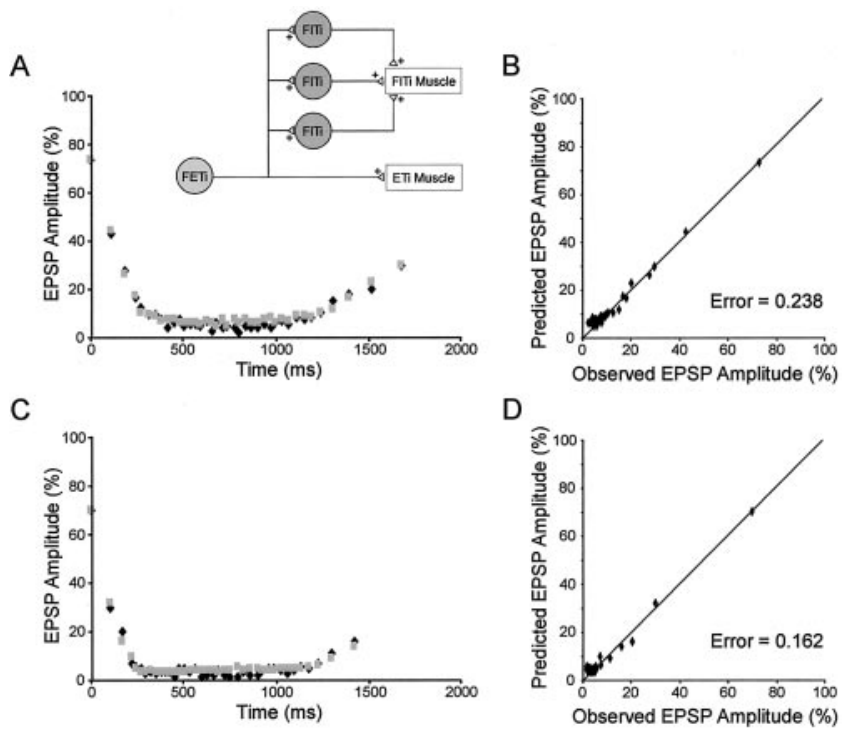

Figure 6. Modeling depression in multiple types of FITi motoneurons. $A$, The model accurately average response of an intermediate FITi motoneuron during FETi spike bursts but with different parameters to the PFFITi motoneuron shown in Figure $4\left(\mathrm{U}=0.72 ; \mathrm{A}=97.04 ; \tau_{\mathrm{Rec}}=\right.$ 729.24). Inset, FETi makes monosynaptic connections to a population of nine FITi motoneurons, up to three of which were recorded sequentially in a single preparation. $B$, Plotting the model against the data show the fit of the model to the data. Points lying on the diagonal indicate a perfect fit. $C$, The fit of the model to a second FFITi motoneuron (anterior position) recorded in the same preparation $\left(U=0.67 ; A=109.11 ; \tau_{\operatorname{Rec}}=508.87\right)$. $D$, As in $B$. 
A

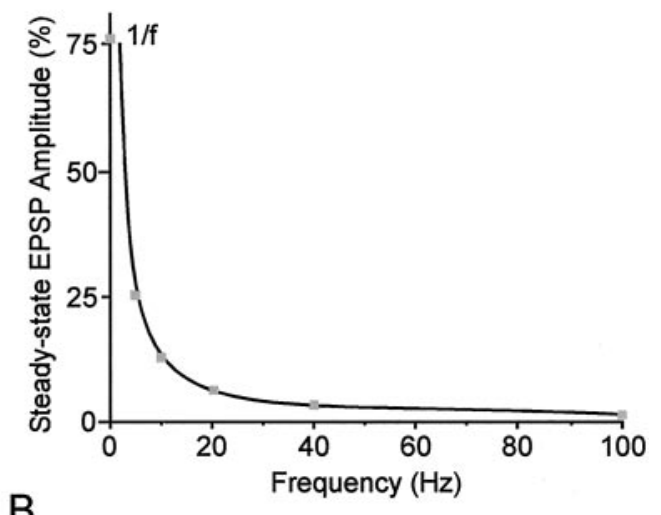

B

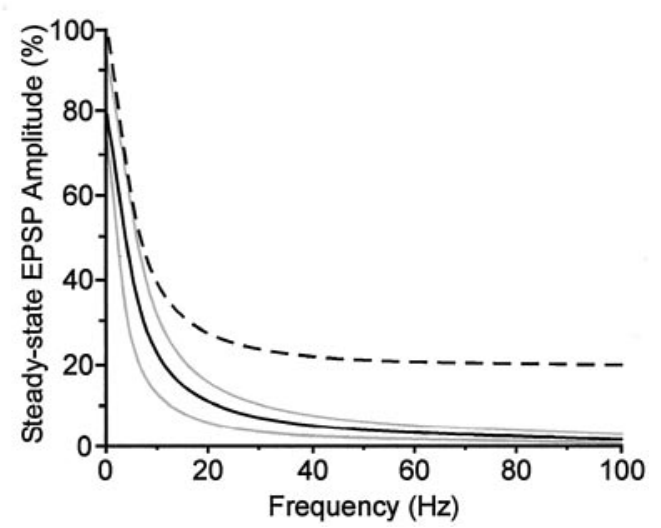

Figure 7. Predictions from the model suggest a behavioral role for depression at the FETiFITi synapse. $A$, Model predictions of steady-state EPSP amplitude fit a $1 / f$ relationship for frequencies above $5 \mathrm{~Hz}$ (see Results for details). B, Model predictions of the steady-state EPSP amplitude for all the FITi motoneurons analyzed. The gray lines indicate the upper and lower limits of the model predictions determined by the parameters for individual FITi motoneurons, and the mean is shown by the bottom black line. The top black is the mean steady-state amplitude for a typical FITi motoneuron during antidromic spike trains.

the width of synaptically evoked spikes had a causal effect on the FlTi EPSP, hyperpolarizing current was injected into FETi before the first spike of a LR-evoked spike train, increasing the spike width. The increased spike width and height produced a concomitant increase in the FlTi EPSP amplitude, implying a causal relationship between the presynaptic waveform and the FlTi EPSP (Fig. 10B). Reduction in the FETi spike width with increasing depolarization suggests that synaptic inputs may be responsible for this spike width modulation. Additionally, reduction in spike width could also be induced during antidromic spike trains by evoking synaptic inputs to FETi, providing evidence that synaptic inputs are sufficient to account for the differences in spike width between LR-evoked and antidromic spikes (Fig. 10C). This reduction in antidromic spike width was accompanied by a concomitant reduction in the amplitude of the FITi EPSP (Fig. 10D), suggesting that the reduction in FETi spike width evoked by synaptic inputs is sufficient to generate the enhanced level of depression at the FETi-FlTi synapse during LR-evoked spike trains.

\section{Discussion}

The importance of short-term depression in synaptic information transfer has been shown both experimentally and theoretically in many systems; yet despite this, the role of short-term depression during synaptically generated natural spike trains re- mains largely unknown. By making use of a circuit that transforms sensory input from a proprioceptor (LR) into a motor output, we have compared short-term depression elicited by synaptically or antidromically driven spike trains at a synapse between two identified motoneurons (FETi-FlTi synapse). The two stimulation regimes produced markedly different dynamics of short-term depression; during LR-evoked spike trains, both the rate and magnitude of depression was greater than during antidromic spike trains at similar spike frequencies. Moreover, the synaptic depression during LR-evoked spike trains could not be estimated from the antidromic, constant frequency spike trains.

Several factors could have contributed to differences between the stimulation regimes, including the spiking statistics, spike waveform, or presence/absence of synaptic inputs. Modeling the depression at FETi-FlTi synapse showed that increased levels of depression during LR-evoked spike trains compared with antidromic spike trains were not because of the different spiking statistics. Additionally, despite differences in the overall shape of the spike waveform, single antidromically or synaptically generated spikes in FETi evoke similar amplitude EPSPs in FlTi motoneurons. This suggested that depolarizing synaptic inputs during LR-evoked spike trains in FETi were responsible for the increased depression at the FETi-FlTi synapse. Depolarizing synaptic inputs to FETi correlated with spike width changes during LR-evoked spike trains that did not occur during antidromic spike trains. Manipulating the FETi spike width showed that it contributed to FITi EPSP amplitude and, therefore, may have contributed to the dynamics of short-term synaptic depression observed in FlTi motor neurons during LR-evoked spike trains.

\section{How do synaptic inputs affect the efficacy of transmission at the FETi-FlTi synapse?}

The presence of depolarizing synaptic inputs during LR-evoked spike trains correlates strongly with a $40 \%$ reduction in the FETi spike width. During constant frequency antidromic spike trains, there is only a $5 \%$ reduction in spike width under physiological conditions, suggesting that reduction in spike width may be responsible for the increased level of depression during LR-evoked spike trains. This is supported by two observations: (1) antidromic spike width is reduced in the presence of depolarizing synaptic inputs; and (2) FlTi EPSP amplitude is increased when FETi spike width is increased by hyperpolarizing current injection. Changes in spike width may affect the presynaptic calcium entry, thereby affecting the amount of neurotransmitter released at a synapse; small increases in spike width $(\sim 20 \%)$ at both cerebellar synapses and in the squid produce large (twofold) increases in the postsynaptic current (Augustine, 1990; Jackson et al., 1991; Sabatini and Regehr, 1997).

Several possible mechanisms could explain the spike width reduction induced by synaptic inputs in FETi. Electron microscopy studies of the FETi-FlTi synapse have revealed that the input and output synapses in FETi are in close proximity in a region of neuropil that lacks voltage-gated $\mathrm{Na}^{+}$channels (Gwilliam and Burrows, 1980; Burrows et al., 1989). Additionally, the spike initiation zone occurs in the region of the primary neurite that bears arborizations containing input synapses, potentially enabling them to modulate spike properties (Gwilliam and Burrows, 1980). This suggests that synaptic inputs reduce the FETi membrane resistance, reducing the membrane time constant throughout the neuropil and in the primary neurite close to the spike initiation zone. Consequently, the width of a spike propagating into this low resistance membrane would be reduced before reaching the output synapses activating voltage-gated $\mathrm{Ca}^{2+}$ 
A
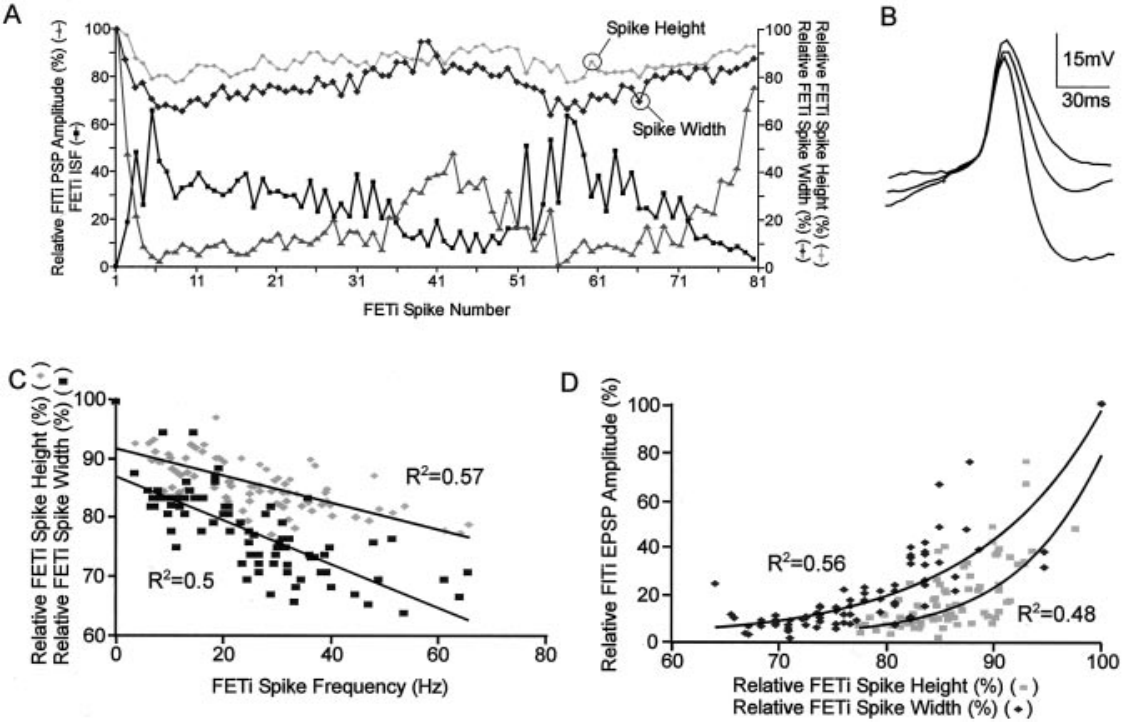

Figure 8. Changes in the presynaptic waveform during natural-like bursts of spikes in FETi. $A$, The spike height and width varied during synaptically evoked bursts of spikes in FETi. $B$, Three FETispikes aligned at their onset show clear changes in both spike height and width. C, Correlation of the FETispike height and width with the frequency of spiking during bursts of spikes as shown in A.D, Correlation of the FITi EPSP amplitude with the FETi spike height and width during synaptically evoked bursts of spikes.
B

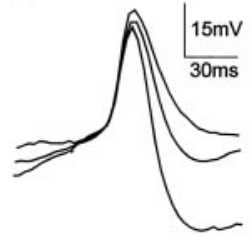

modulators on presynaptic calcium channels (Yawo and Chuhma, 1993; Scanziani et al., 1997) may all contribute to the observed depression.

At the FETi-FlTi synapse, the depletion model accounts for several features of depression during antidromic spike trains, although it cannot account for the inability to produce depression below 20\% despite a fivefold increase in spike frequency (Parker, 1995). In contrast, there is no such limit on the steady-state level of depression during LR-evoked spike trains. However, depression during LR-evoked spike trains is unlikely to fit the depletion model because during depression there are changes in the presynaptic waveform. The reduction in both FETi spike width and height during LR-evoked spike trains produces a concomitant reduction in the FlTi EPSP amplitude, possibly because of a reduction in the levels of presynaptic calcium (Augustine, 1990; Jackson et al., 1991; Sabatini and Re- channels for shorter periods of time, leading to smaller FlTi EPSPs. In addition, increasing numbers of depolarizing inputs evoked by LR stimulation would increase the FETi spike frequency and further reduce the spike width. This suggests that at the FETi-FlTi synapse presynaptic inputs reduce the spike waveform, thereby contributing to the dynamics of short-term depression.

Presynaptic inputs may affect synaptic output via changes in the spike waveform in both vertebrate and invertebrate neural networks (Nusbaum et al., 1997; Rudomin et al., 1998). For example, at afferent terminals in arthropods, shunting is primarily responsible for inducing changes in the presynaptic waveform that correlate with reduced postsynaptic responses (Cattaert et al., 1992; Burrows and Matheson, 1994; Cattaert and El Manira, 1999). Additionally, neurons in which graded membrane potentials reach output synapses, the presynaptic membrane potential may affect the PSP amplitude. For example, at inhibitory interneuron synapses (heart interneuron-heart excitor motoneuron) in the leech, presynaptic membrane potential affects the amplitude of PSPs (Nicholls and Wallace, 1978). The interaction of presynaptic inputs with the dynamics of short-term depression provides an additional level of plasticity for information processing in neural networks. Potentially presynaptic inputs could tune the dynamics of short-term depression or facilitation by modulating the shape of the spike waveform, enabling information to be transmitted not only by spike rate or interspike interval but also by spike width or spike height.

\section{Mechanisms underlying depression and recovery at the FETi-FlTi synapse}

Depletion of the releasable transmitter store explains many of the features of short-term depression at several synapses (Liley and North, 1953; Elmqvist and Quastel, 1965; Rosenthal, 1969; Kusano and Landau, 1975), however, at other synapses, changes in the presynaptic spike waveform (Dunlap and Fischbach, 1978; Klein and Kandel, 1980; Augustine, 1990; Sabatini and Regehr, 1997), inactivation of the presynaptic calcium current (Forsythe et al., 1998; Patil et al., 1998), or the inhibitory action of neuro-
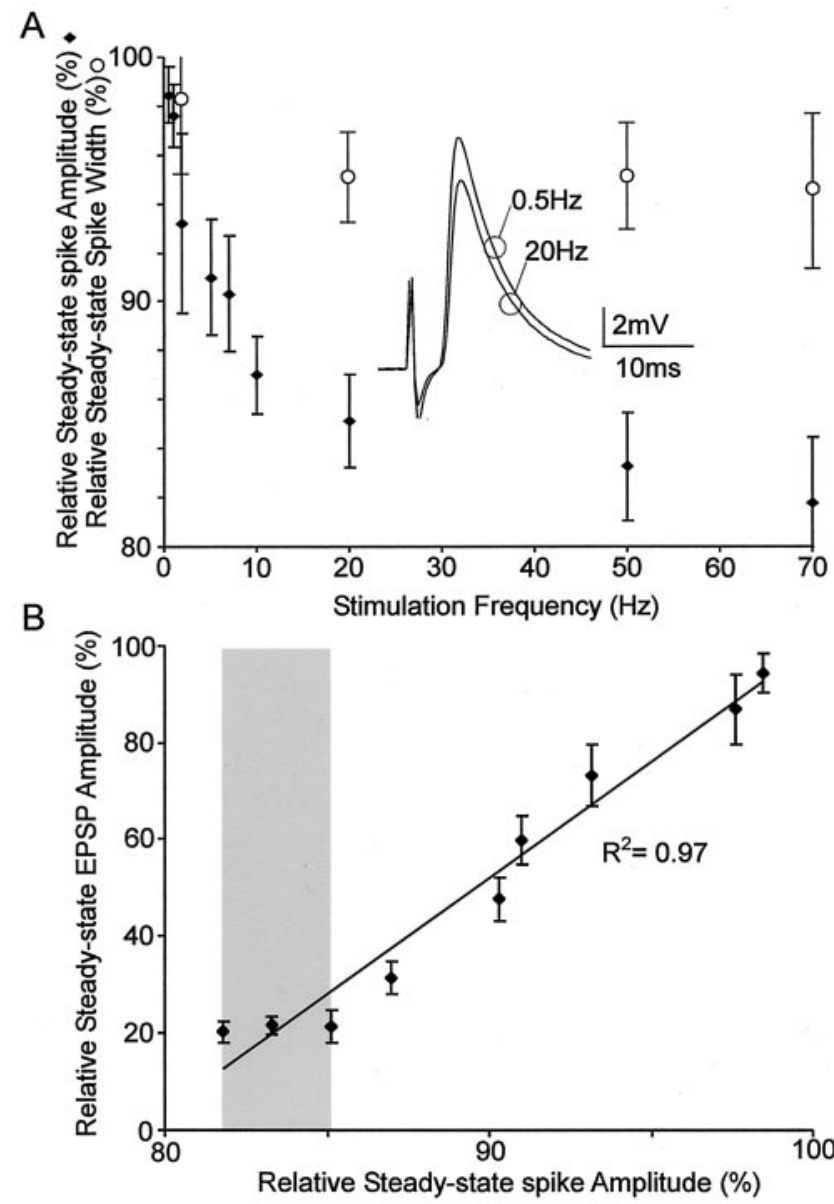

Figure 9. Changes in the presynaptic waveform during antidromically generated spike trains in FETi. $A$, The steady-state spike height (black diamonds, calculated after the initial 10 spikes) and steady-state spike width (open circles) plotted against the antidromic spike frequency. Inset, The steady-state spike heights at 0.5 and $20 \mathrm{~Hz}$. B, Relationship between the steady-state EPSP amplitude and the steady-state spike amplitude. The shaded region shows the plateau in the FITi EPSP amplitude despite a continued drop in the FETi spike amplitude. 
gehr, 1997). Depression at the FETiFlTi synapse occurs during antidromic spike trains in the absence of presynaptic waveform changes, suggesting that during LR-evoked spike trains several mechanisms combine to produce the observed depression.

\section{Behavioral significance of depression at the FETi-FlTi synapse}

The central synaptic connection between FETi and the FlTi motoneurons is thought to be a specialization for the generation of the jumping and kicking motor programs; FETi is usually activated only during kicking or jumping at the same time as the antagonistic FlTi motoneurons are active (Burrows, 1996), whereas during walking antagonistic tibial muscles are not coactivated and spikes are rarely observed in FETi (Burns and Usherwood, 1979). Although the FETi-FlTi synapse was originally postulated to increase FlTi muscle tension during the co-contraction phase of the kick (Heitler and Burrows, 1977), the rapid depression of this synaptic connection during antidromic spike trains suggested that inputs from this synapse could not be solely responsible for maintaining FlTi muscle tension (Burrows et al., 1989; Parker, 1995). Yet, the residual FlTi EPSP amplitude of $20 \%$ during antidromic spike trains of up to $100 \mathrm{~Hz}$ suggests that even after maximum depression inputs from FETi would still contribute to the FlTi spike frequency. This clearly is not the case during LR-evoked spike trains, during which the FlTi EPSP depresses rapidly to just $5 \%$ of its initial amplitude. The increase in both the rate and magnitude of the depression at the FETi-FlTi synapse are reflected in the model. The $U$ parameter and the limiting frequency determine whether the FlTi postsynaptic response reflects the rate of spiking or the onset of spiking in FETi. $U$ is the fraction of neurotransmitter used after a spike, hence, high values of $U$ will cause the synapse to depress rapidly to its steady-state amplitude (Tsodyks and Markram, 1997). The limiting frequency sets the upper limit to the frequency range over which the synapse can transmit information about the presynaptic spike rate; above the limiting frequency, synapses mainly transmit temporal information. The high $U$ parameter at the FETi-FlTi synapse was consistent between synaptic connections in different individuals (0.52-0.89). Coupled with the high spike frequencies during LR-evoked spike trains (up to $70 \mathrm{~Hz}$ ) and the low-limiting frequency $(\sim 5 \mathrm{~Hz})$, this suggests that the FETi-FlTi synapse may be crucial for signaling the onset of FETi motoneuron activity at the start of co-contraction rather than contributing to the spike rate of the FlTi motoneurons during co-contraction.

Depression of the FETi-FITi synapse may allow FETi activity to contribute to FlTi activity at the onset of co-contraction but enable uncoupling of their activity later in co-contraction, allowing other inputs to determine the pattern of FlTi activity. It may also enable FETi to continue to spike during the period of FlTi motoneuron inhibition that precedes tibial release without exciting the FlTi motoneurons, thus preventing contraction of the FlTi muscle that would reduce the velocity of the tibia once released.
B

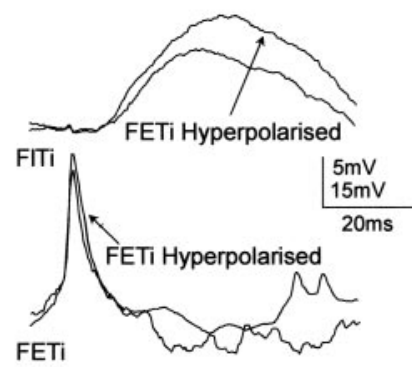

D

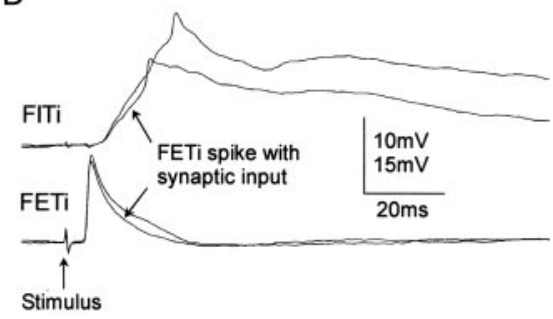

\section{References}

Abbott LF, Sen K, Varela JA, Nelson SB (1997) Synaptic depression and cortical gain control. Science 275:220-222.

Augustine G (1990) Regulation of neurotransmitter release at the squid giant synapse by presynaptic delayed rectifier current. J Physiol (Lond) 431:343-364.

Burns MD, Usherwood PNR (1979) The control of walking in Orthoptera. II. Motor neurone activity in normal free-walking animals. J Exp Biol 79:69-98.

Burrows M (1995) Motor patterns during kicking movements in the locust. J Comp Physiol [A] 176:289-305.

Burrows M (1996) The neurobiology of an insect brain, p 682. Oxford: Oxford University.

Burrows M, Matheson T (1994) A presynaptic gain control mechanism among sensory neurons of a locust leg proprioceptor. J Neurosci 14:272-282.

Burrows M, Watson ADH, Brunn DE (1989) Physiological and ultrastructural characterisation of a central synaptic connection between identified motor neurons in the locust. Eur J Neurosci 1:111-126.

Cattaert D, El Manira A (1999) Shunting versus inactivation: analysis of presynaptic inhibitory mechanisms in primary afferents of the crayfish. J Neurosci 19:6079-6089.

Cattaert D, El Manira A, Clarac F (1992) Direct evidence for presynaptic inhibitory mechanisms in crayfish sensory afferents. J Neurosci 67:610-624.

Combes D, Meyrand P, Simmers J (1999) Dynamic restructuring of a rhythmic motor program by a single mechanoreceptor neuron in lobster. J Neurosci 19:3620-3628.

Del Castillo J, Katz B (1954) Statistical factors involved in neuromuscular facilitation and depression. J Physiol (Lond) 124:574-585.

Dunlap K, Fischbach GD (1978) Neurotransmitters decrease the calcium component of sensory neurone action potentials. Nature 276:837-839.

Eccles JC, Katz B, Kuffler SW (1941) Nature of the "endplate potential" in curarized muscle. J Neurophysiol 4:362-387.

Elmqvist D, Quastel DMJ (1965) A quantitive study of end-plate potentials in isolated human muscle. J Physiol (Lond) 178:505-529.

Feng TP (1941) Studies on the neuromuscular junction. Chin J Physiol 16:341-372. 
Finnerty GT, Roberts LSE, Connors BW (1999) Sensory experience modifies the short-term dynamics of neocortical synapses. Nature 400:367-371.

Forsythe ID, Tsujimoto T, Barnes-Davies M, Cuttle MF, Takahashi T (1998) Inactivation of presynaptic calcium current contributes to synaptic depression at a fast central synapse. Neuron 20:797-807.

Gwilliam GF, Burrows M (1980) Electrical characteristics of the membrane of an identified insect motor neurone. J Exp Biol 86:49-61.

Heitler WJ, Burrows M (1977) The locust jump. II. Neural circuits of the motor program. J Exp Biol 66:221-241.

Hoyle G, Burrows M (1973) Neural mechanisms underlying behaviour in the locust Schistocerca gregaria. I. Physiology of identified motorneurons in the metathoracic ganglion. J Neurobiol 4:23-41.

Jackson MB, Konnerth A, Augustine G (1991) Action potential broadening and frequency-dependent facilitation of calcium signals in pituitary nerve terminals. Proc Natl Acad Sci USA 88:380-384.

Klein M, Kandel ER (1980) Mechanism of calcium current modulation underlying presynaptic facilitation and behavioral sensitization in Aplysia. Proc Natl Acad Sci USA 77:6912-6916.

Kusano K, Landau EM (1975) Depression and recovery of transmission at the squid giant synapse. J Neurosci 4:125-130.

Liley AW, North KAK (1953) An electrical investigation of effects of repetitive stimulation on mammalian neuromuscular junction. J Neurophysiol 16:509-527.

Markram H, Wang Y, Tsodyks M (1998) Differential signalling via the same axon of neocortical pyramidal neurons. Proc Natl Acad Sci USA 95:5323-5328.

Nicholls J, Wallace BG (1978) Modulation of transmission at an inhibitory synapse in the central nervous system of the leech. J Physiol (Lond) 281:157-170.

Nusbaum MP, El Manira A, Gossard JP, Rossignol S (1997) Presynaptic mechanisms during rhythmic activity in vertebrates and invertebrates. In: Neurons, networks and motor behavior (Stein PSG, Grillner S, Selverston AI, Stuart DG, eds), pp 237-253. Cambridge, MA: Massachusetts Institute of Technology.

Parker D (1995) Depression of synaptic connections between identified motor neurons in the locust. J Neurophysiol 74:529-538.
Parker D (2000) Spinal-cord plasticity. Mol Neurobiol 22:55-80.

Patil PG, Brody DL, Yue DT (1998) Preferential closed-state inactivation of neuronal calcium channels. Neuron 20:1027-1038.

Rosenthal J (1969) Post-tetanic potentiation at the neuromuscular junction of the frog. J Physiol (Lond) 203:121-133.

Rudomin P, Romo R, Mendell LM (1998) Presynaptic inhibition and neural networks. New York: Oxford UP.

Sabatini BL, Regehr WG (1997) Control of neurotransmitter release by pre synaptic waveform at the granule cell to purkinje cell synapse. J Neurosci 17:3425-3435.

Scanziani M, Salin PA, Vogt KE, Malenka RC, Nicoll RA (1997) Usedependent increases in glutamate concentration activate presynaptic metabotropic glutamate receptors. Nature 385:630-634.

Sen K, Jorge-Rivera JC, Marder E, Abbott LF (1996) Decoding synapses J Neurosci 16:6307-6318.

Sieglebaum SA, Camardo JS, Kandel ER (1982) Serotonin and cyclic AMP close single $\mathrm{K}^{+}$channels in Aplysia sensory neurons. Nature 299:413-417.

Thomson AM, Deuchars J (1994) Temporal and spatial properties of local circuits in neocortex. Trends Neurosci 16:222-226.

Tsodyks MV, Markram H (1997) The neural code between neocortical pyramidal neurons depends on neurotransmitter release probability. Proc Natl Acad Sci USA 94:719-723.

Usherwood PNR, Grundfest H (1965) Peripheral inhibition of skeletal muscle of insects. J Neurophysiol 28:497-518.

Watson ADH, Burrows M (1981) Input and output synapses on identified motor neurones of a locust revealed by the intracellular injection of horseradish peroxidase. Cell Tissue Res 215:325-332.

Wheeler DB, Randall A, Tsien RW (1996) Changes in action potential duration alter reliance of excitatory synaptic transmission on multiple types of $\mathrm{Ca}^{2+}$ channels in rat hippocampus. J Neurosci 14:5613-5622.

Yawo H, Chuhma N (1993) Preferential inhibition of $\omega$-conotoxinsensitive presynaptic $\mathrm{Ca}^{2+}$ channels by adenosine autoreceptors. Nature 365:256-258.

Zar JH (1996) Biostatistical analysis. Upper Saddle River, NJ: Prentice-Hall, Inc.

Zucker RS (1989) Short-term synaptic plasticity. Annu Rev Neurosci 12:13 31. .0 